\title{
TWO DETECTION RESULTS OF KHOVANOV HOMOLOGY ON LINKS
}

\author{
ZHENKUN LI, YI XIE, AND BOYU ZHANG
}

\begin{abstract}
We prove that Khovanov homology with $\mathbb{Z} / 2$-coefficients detects the link L7n1, and the union of a trefoil and its meridian.
\end{abstract}

\section{INTRODUCTION}

Given an oriented link $L$ in $S^{3}$ and a commutative ring $R$, Khovanov homology [Kho00] assigns a bi-graded $R$-module $\mathrm{Kh}(L ; R)$ to the link $L$. In 2011, Kronheimer and Mrowka [KM11] proved that Khovanov homology detects the unknot. Since then, many other detection results of Khovanov homology have been obtained. It is now known that Khovanov homology detects the unlink [BS15, HN13], the trefoil [BS18], the Hopf link [BSX19], the forest of unknots [XZ19], the splitting of links [LS19], and the torus link $T(2,6)$ [Mar20].

In [XZ20], a classification is given for all links $L$ such that $\operatorname{rank}_{\mathbb{Z} / 2} \mathrm{Kh}(L ; \mathbb{Z} / 2) \leq 8$ and all 3-component links $L$ such that $\operatorname{rank}_{\mathbb{Z} / 2} \operatorname{Kh}(L ; \mathbb{Z} / 2) \leq 12$. By [Shu14, Corollary 3.2.C], $\operatorname{rank}_{\mathbb{Z} / 2} \operatorname{Kh}(L ; \mathbb{Z} / 2)=2 \operatorname{rank}_{\mathbb{Z} / 2} \operatorname{Khr}(L ; \mathbb{Z} / 2)$, where $\mathrm{Khr}$ denotes the reduced Khovanov homology. Moreover, the parity of $\operatorname{rank}_{\mathbb{Z} / 2} \operatorname{Khr}(L ; \mathbb{Z} / 2)$ is invariant under crossing changes and hence is always even for 2-component links (as is the case for the 2 -component unlink). Therefore $\operatorname{rank}_{\mathbb{Z} / 2} \mathrm{Kh}(L ; \mathbb{Z} / 2)$ is always a multiple of 4 . As a consequence, if a 2 -component link $L$ satisfies $\operatorname{rank}_{\mathbb{Z} / 2} \operatorname{Kh}(L ; \mathbb{Z} / 2)>8$, then $\operatorname{rank}_{\mathbb{Z} / 2} \operatorname{Kh}(L ; \mathbb{Z} / 2) \geq 12$.

This paper studies 2 -component links $L$ such that $\operatorname{rank}_{\mathbb{Z} / 2}(L ; \mathbb{Z} / 2)=12$. Among 2-component links with crossing numbers less than or equal to 7 , there are four links (up to mirror images) satisfying $\operatorname{rank}_{\mathbb{Z} / 2}(L ; \mathbb{Z} / 2)=12$. These links are:

(1) L7n1 in the Thistlethwaite Link Table,

(2) L6a3 in the Thistlethwaite Link Table,

(3) the disjoint union of a trefoil and an unknot,

(4) the union of a trefoil and its meridian.

Question 1.1. Suppose $L$ is a 2-component link with $\operatorname{rank}_{\mathbb{Z} / 2}(L ; \mathbb{Z} / 2)=12$, is it true that $L$ must be isotopic (up to mirror image) to one of the links listed above?

Instead of giving a full answer to the question above, we show that Khovanov homology (with the bi-grading) detects the link L7n1, and the union of a trefoil with its meridian, from the list above. Since [XZ19] proved that Khovanov homology detects the disjoint union of a trefoil and an unknot, and Martin [Mar20] recently proved that Khovanov homology detects L6a3, we conclude that Khovanov homology detects all the links on the list.

In the following, we will call the link L7n1 as $L_{1}$, and the union of a trefoil with a meridian $L_{2}$. Moreover, we fix the chirality and orientation of these two links by 


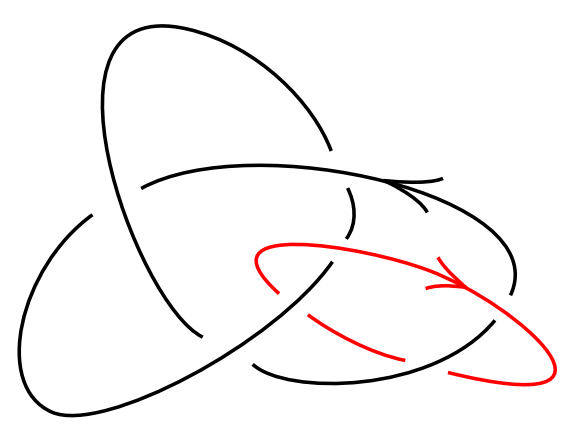

$L_{1}=\mathrm{L} 7 \mathrm{n} 1$

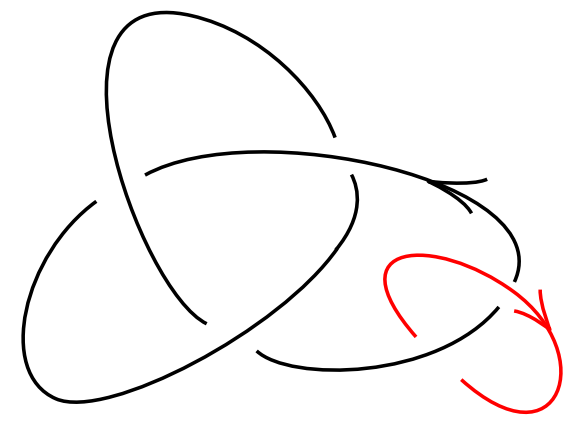

$L_{2}=$ trefoil $\cup$ meridian

Figure 1. The two links $L_{1}$ and $L_{2}$

Figure 1. Notice that the link $L_{1}$ can also be described as the closure of the 2-braid $\sigma_{1}^{3}$ together with an axis unknot.

Recall that the internal grading of Khovanov homology is defined by $h-q$ in [BS15], where $h$ is the homological grading and $q$ is the quantum grading. The precise statement of our detection result is given as follows.

Theorem 1.2. Let $L_{1}, L_{2} \subset S^{3}$ be the oriented links as shown in Figure 1, and let $i \in\{1,2\}$. Suppose $L \subset S^{3}$ is a 2-component oriented link, such that

$$
\mathrm{Kh}(L ; \mathbb{Z} / 2) \cong \mathrm{Kh}\left(L_{i} ; \mathbb{Z} / 2\right)
$$

as abelian groups equipped with the internal gradings, then $L$ is isotopic to $L_{i}$ as oriented links.

The proof of Theorem 1.2 depends on a rank inequality between reduced Khovanov homology and knot Floer homology by Dowlin [Dow18], and a braid detection property of link Floer homology by Martin [Mar20]. The main ingredient of the proof of Theorem 1.2 is the following proposition, which is established in Section 3.

Proposition 1.3. Let $L=K \cup U$ be a link such that $U$ is an unknot and $K$ is either an unknot or a trefoil. Let $l=|\operatorname{lk}(K, U)|$ be the linking number of $K$ and $U$. Suppose $l>0$, and

$$
\operatorname{dim}_{\mathbb{Q}} \widehat{\operatorname{HFK}}(L ; \mathbb{Q}) \leq 12,
$$

where $\widehat{\mathrm{HFK}}$ is the knot Floer homology defined in [Ras03, OS04]. Then at least one of the following holds:

(1) $K$ is the closure of an l-braid with axis $U$.

(2) $l=1, K$ is an unknot.

(3) $l=1, K$ is a trefoil, $U$ is the meridian of $K$.

Recall that a 2-component link $K_{1} \cup K_{2}$ is said to be exchangeably braided (or mutually braided) if both $K_{1}$ and $K_{2}$ are unknots, $K_{1}$ is a braid closure with axis $K_{2}$, and $K_{2}$ is a braid closure with axis $K_{1}$. The concept of exchangeably braided links was introduced and studied by Morton [Mor85]. We will also need the following result from $[\mathrm{XZ20}]$. 
Proposition 1.4 ([XZ20, Corollary 3.9]). Suppose $L$ is an exchangeably braided link with linking number $l \geq 3$, then we have $\operatorname{rank}_{\mathbb{Q}} \widehat{\operatorname{HFK}}(L ; \mathbb{Q}) \geq 12$. Moreover, if $l>3$, then $\operatorname{rank}_{\mathbb{Q}} \widehat{\operatorname{HFK}}(L ; \mathbb{Q})>12$.

We will prove Theorem 1.2 in Section 4 as a consequence of Proposition 1.3, Proposition 1.4, Dowlin's rank inequality [Dow18, Corollary 1.7], and Batson-Seed's spectral sequence [BS15].

Acknowledgement. The first author is supported by his advisor Tom Mrowka's NSF Grant 1808794.

\section{LiNK FloeR HOMOLOGY}

This section reviews the basic properties of link Floer homology and proves a result on the rank of link Floer homology that will play an important role in the proof of Proposition 1.3.

The link Floer homology was originally defined for $\mathbb{Z} / 2$-coefficients by Ozsváth and Szabó in [OS04], and was generalized to $\mathbb{Z}$-coefficients in [Sar11]. We will work with $\mathbb{Q}$-coefficients in order to invoke Dowlin's spectral sequence [Dow18]. For the rest of this section, all Floer homology groups are with $\mathbb{Q}$-coefficients and it will be omitted from the notation.

Given an oriented $n$-component link $L \subset S^{3}$, its link Floer homology $\widehat{\operatorname{HFL}}(L)$ carries a homological grading over $\mathbb{Z}$ and $n$ Alexander gradings associated to the $n$ components of $L$. The Alexander grading associated to the $i$-th component $K_{i}$ takes values in either $\mathbb{Z}$ or $\mathbb{Z}+\frac{1}{2}$, which depends on the parity of the linking number $\operatorname{lk}\left(K_{i}, L-K_{i}\right)$.

By [OS08a], when $n \geq 2$, the link Floer homology recovers the multi-variable Alexander polynomial in the following sense:

$$
\begin{aligned}
& \sum_{a_{1}, \cdots, a_{n}} \chi\left(\widehat{\operatorname{HFL}}\left(L, a_{1}, \cdots, a_{n}\right)\right) \cdot T_{1}^{a_{1}} \cdots T_{n}^{a_{n}} \\
& \doteq\left(T_{1}^{1 / 2}-T_{1}^{-1 / 2}\right) \cdots\left(T_{n}^{1 / 2}-T_{n}^{-1 / 2}\right) \Delta_{L}\left(T_{1}, \cdots, T_{n}\right),
\end{aligned}
$$

where $\widehat{\mathrm{HFL}}\left(L, a_{1}, \cdots, a_{n}\right)$ is the component of $\widehat{\mathrm{HFL}}(L)$ with multi-Alexander grading $\left(a_{1}, \cdots, a_{n}\right)$, and $\chi(\cdot)$ denotes the Euler characteristic with respect to the homological grading. The notation "" means that the two sides are equal up to a multiplication by $\pm T_{1}^{b_{1}} \cdots T_{n}^{b_{n}}$ for some $b_{1}, \cdots b_{n} \in \frac{1}{2} \mathbb{Z}$. There is also a symmetry

$$
\widehat{\operatorname{HFL}}\left(L, a_{1}, \cdots, a_{n}\right) \cong \widehat{\operatorname{HFL}}\left(L,-a_{1}, \cdots,-a_{n}\right) .
$$

The following proposition is a special case of the Thurston norm detection property of link Floer homology.

Proposition 2.1 ([OS08b, Theorem 1.1]). Suppose $L=K \cup U \subset S^{3}$ is a 2component link with an unknotted component $U$ and $l=|\operatorname{lk}(K, U)|>0$. Then the top Alexander grading of $\widehat{\mathrm{HFL}}(L)$ associated to $U$ is $\frac{l}{2}$ if and only if $U$ has a Seifert disk that intersects $K$ transversely at $l$ points.

Remark 2.2. The proof of [OS08b, Theorem 1.1] was originally given for $\mathbb{Z} / 2$ coefficients, but the same argument applies to $\mathbb{Q}$-coefficients. Alternatively, a similar norm-detection property for instanton Floer homology was established by [GL19] 
using sutured manifold decompositions and the formal properties of Floer homology, and the same argument can be carried over to Heegaard Floer homology with Q-coefficients.

The following is a weaker version of a result from [Mar20].

Proposition 2.3 ([Mar20, Corollary 2]). Let $L=K \cup U \subset S^{3}$ be a 2-component link such that $U$ an unknot and $l=|\operatorname{lk}(K, U)|>0$. Then $K$ is the closure of a braid with axis $U$ if and only if the dimension of $\widehat{\operatorname{HFL}}(L)$ is 2 at the top Alexander grading associated to $U$.

The link Floer homology $\widehat{\mathrm{HFL}}$ can be interpreted by sutured Floer homology using the following proposition. Here we use SFH to denote the sutured Floer homology defined by Juhász in [Juh06].

Proposition 2.4 ([Juh06, Proposition 9.2]). Suppose $L=K_{1} \cup \ldots \cup K_{n}$ is an oriented link, and let $S^{3}-N(L)$ be the link complement. Let $\gamma$ be a suture on $\partial\left(S^{3}-\right.$ $N(L)$ ) which consists of two meridians of each $K_{i}$. Then there is an isomorphism

$$
\widehat{\mathrm{HFL}}(L) \cong \operatorname{SFH}\left(S^{3}-N(L), \gamma\right) \text {. }
$$

Moreover, the Alexander grading associated to $K_{i}$ corresponds to the grading induced by a Seifert surface of $K_{i}$ on $\operatorname{SFH}\left(S^{3}-N(L), \gamma\right)$.

Remark 2.5. The original statement is for $\mathbb{Z} / 2$-coefficients, but the proof is done by examining the Heegaard diagrams, which also works for $\mathbb{Q}$-coefficients.

We also need the following proposition from [Juh10].

Proposition 2.6 ([Juh10, Proposition 9.2]). Suppose $(M, \gamma)$ is a balanced sutured manifold. Suppose $\gamma_{0}$ is a component of $\gamma$ that is homologically essential on $\partial M$. Let $\gamma^{\prime}$ be a suture on $\partial M$ obtained by adding two parallel copies of $\gamma_{0}$ to $\gamma$. Then we have

$$
\operatorname{SFH}\left(M, \gamma^{\prime}\right) \cong \operatorname{SFH}(M, \gamma) \otimes_{\mathbb{Q}} \mathbb{Q}^{2} .
$$

The main result of this section is the following proposition.

Proposition 2.7. Suppose $L=K \cup U \subset S^{3}$ is a 2-component link with an unknotted component $U$ and $l=|\operatorname{lk}(K, U)|>0$, and suppose $U$ has a Seifert disk $D$ that intersects $K$ transversely at $l$ points. Then

$$
\operatorname{dim}_{\mathbb{Q}} \widehat{\operatorname{HFL}}\left(L, \frac{l}{2}\right) \equiv 2 \bmod 4,
$$

where $\widehat{\mathrm{HFL}}(L, l / 2)$ is the component of $\widehat{\mathrm{HFL}}(L)$ with degree $l / 2$ on the Alexander grading associated to $U$.

In order to prove Proposition 2.7, we need to establish the following property of sutured Floer homology.

Proposition 2.8. Let $l \in \mathbb{Z}^{+}$, let $T \subset[-1,1] \times D^{2}$ be a tangle given by $T=$ $\alpha_{1} \cup \cdots \cup \alpha_{l}$, where $\alpha_{i}$ is an arc connecting $\{-1\} \times D^{2}$ and $\{1\} \times D^{2}$ for all $i$. Let $M_{T}=[-1,1] \times D^{2}-N(T)$, let $\gamma_{T} \subset \partial M_{T}$ be a suture on $M_{T}$ with $(l+1)$ components: one meridian component on each one of $\partial N\left(\alpha_{1}\right), \cdots \partial N\left(\alpha_{l}\right)$, and a component on $[-1,1] \times \partial D^{2}$ given by $\{\mathrm{pt}\} \times \partial D^{2}$. Then $\operatorname{dim}_{\mathbb{Q}} \operatorname{SFH}\left(M_{T}, \gamma_{T}\right)$ is odd.

We start the proof of Proposition 2.8 by verifying the trivial case. 

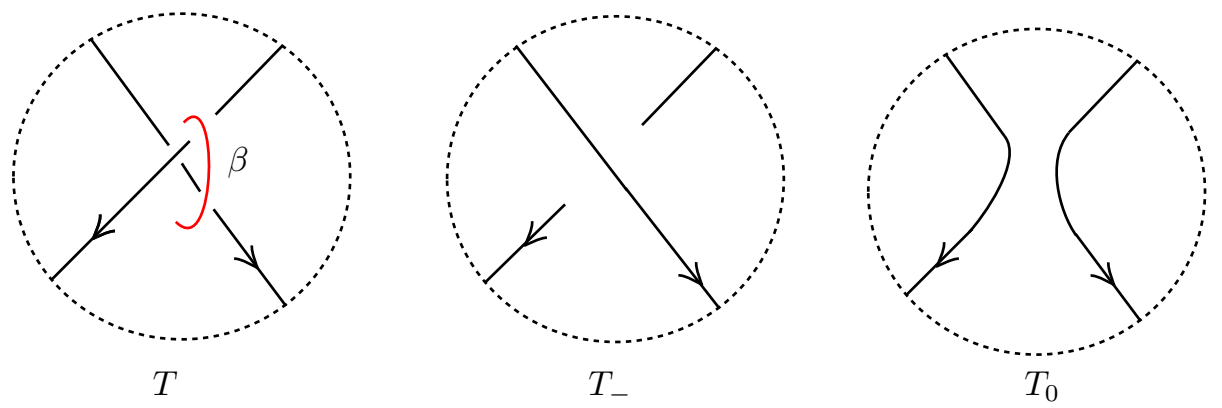

FiguRE 2. Surgery along $\beta$

Lemma 2.9. If $T$ is a product tangle, i.e., there are points $p_{1}, \ldots, p_{n} \subset \operatorname{int}\left(D^{2}\right)$ so that $\alpha_{i}=[-1,1] \times\left\{p_{i}\right\}$ for all $i$, then $\operatorname{dim}_{\mathbb{Q}} \operatorname{SFH}\left(M_{T}, \gamma_{T}\right)$ is odd.

Proof. When $T$ is a product tangle, $\left(M_{T}, \gamma_{T}\right)$ is a product sutured manifold. Hence it follows from [Juh06] that the dimension of $\operatorname{SFH}\left(M_{T}, \gamma_{T}\right)$ is one.

Let $T$ be the tangle in Proposition 2.8. Orient $T$ so that each $\alpha_{i}$ goes from $\{-1\} \times D^{2}$ to $\{1\} \times D^{2}$. Fix a diagram on $[-1,1] \times[-1,1]$ that represents the tangle $T$. We will also denote the diagram by $T$ when there is no source of confusion. For a positive crossing of $T$, we can perform surgeries along the curve $\beta$ as depicted in Figure 2. Let $M_{T,-1}$ be the manifold obtained by performing the (-1)-surgery along $\beta$, and let $M_{T, 0}$ be the manifold obtained by performing the 0 -surgery along $\beta$. Let $T_{-}$be the tangle that only differs from $T$ at the crossing linked by $\beta$ as depicted in Figure 2. It straightforward to show that $\left(M_{T,-1}, \gamma_{T}\right) \cong\left(M_{T_{-}}, \gamma_{T_{-}}\right)$.

Definition 2.10. We call the operation of switching from $T$ to $T_{-}$or from $T_{-}$to $T$ a crossing change.

Lemma 2.11. For any vertical tangle $T \subset[-1,1] \times D^{2}$, there is a finite sequence of crossing changes that takes $T$ to the product tangle.

Now we study the sutured manifold $\left(M_{T, 0}, \gamma_{T}\right)$. Inside $[-1,1] \times D^{2}$, the circle $\beta$ bounds a disk $D$ that intersects the tangle $T$ twice. After performing the 0 -surgery, the boundary $\partial D$ can be capped by a meridian disk in the surgery solid torus, and hence we obtain a 2 -sphere $S$ that intersects the tangle $T$ twice. The intersection of $S$ and $M_{T, 0}$ is a properly embedded annulus $A_{\beta} \subset M_{T, 0}$. We can pick the suture $\gamma_{T}$ so that one boundary component of $A_{\beta}$ lies in $R_{+}\left(\gamma_{T}\right)$ and the other lies in $R_{-}\left(\gamma_{T}\right)$. Then there is a sutured manifold decomposition

$$
\left(M_{T, 0}, \gamma_{T}\right) \stackrel{A_{\beta}}{\rightsquigarrow}\left(M^{\prime}, \gamma^{\prime}\right) .
$$

From [KM10, Section 3.1], we know that $M^{\prime} \cong M_{T_{0}}:=[-1,1] \times D^{2}-N\left(T_{0}\right)$, where $T_{0}$ is another tangle on $[-1,1] \times D^{2}$, possibly having closed components, such that $T_{0}$ only differs from $T$ near the crossing linked by $\beta$ as depicted in Figure 2.

Definition 2.12. We say that $T_{0}$ is obtained from $T_{+}$by an oriented smoothing.

Lemma 2.13. We have

$$
\operatorname{SFH}\left(M_{T, 0}, \gamma_{T}\right) \cong \operatorname{SFH}\left(M_{T_{0}}, \gamma^{\prime}\right),
$$

and $\operatorname{dim}_{\mathbb{Q}} \operatorname{SFH}\left(M_{T_{0}}, \gamma^{\prime}\right)$ is even. 

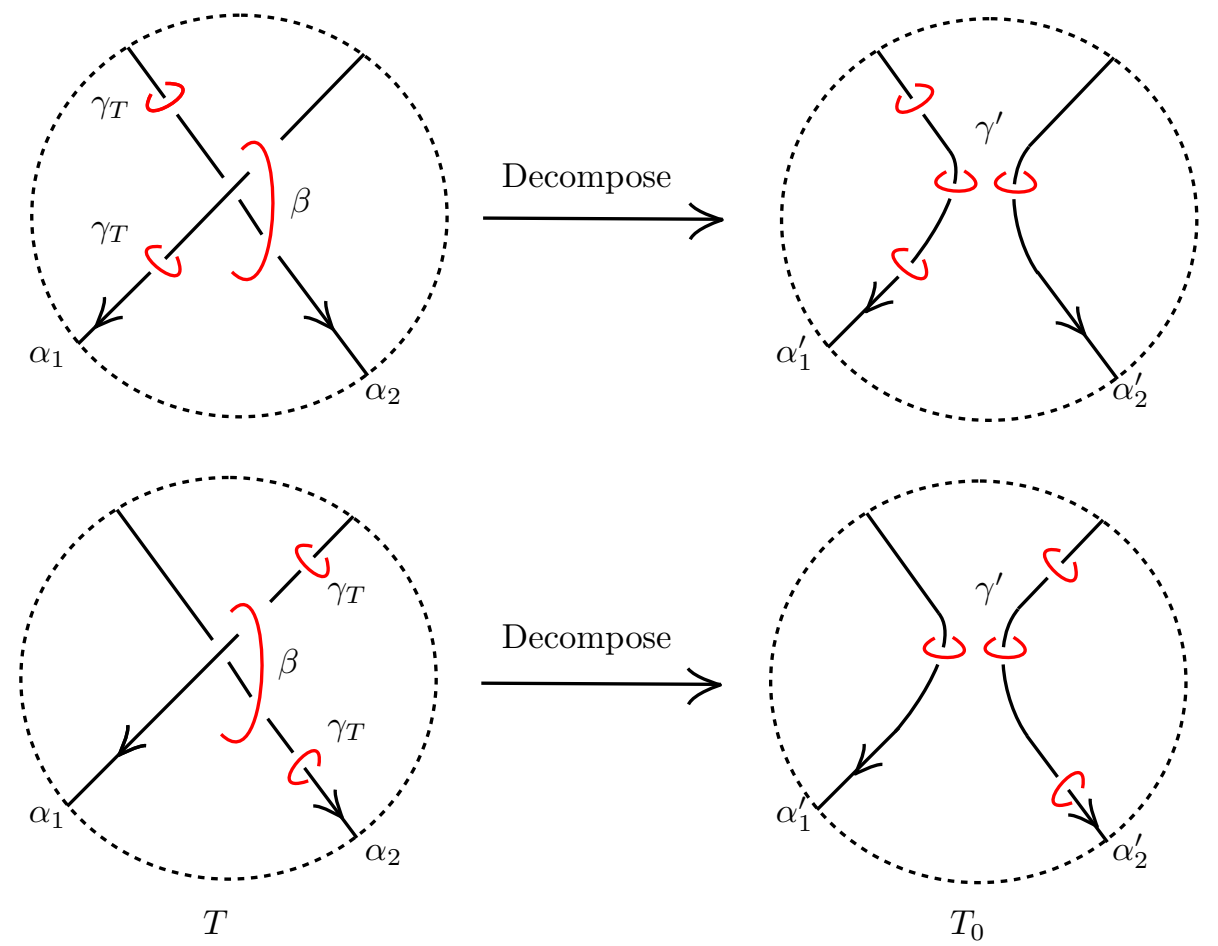

FIGURE 3. Oriented smoothing

Proof. The isomorphism

$$
\operatorname{SFH}\left(M_{T, 0}, \gamma_{T}\right) \cong \operatorname{SFH}\left(M_{T_{0}}, \gamma^{\prime}\right)
$$

follows from [Juh08, Lemma 8.9]. For the parity statement, we argue in two cases.

Case 1. The crossing linked by $\beta$ involves two different components of $T$. Without loss of generality we can assume that they are $\alpha_{1}$ and $\alpha_{2}$. See Figure 3. Recall we have an annulus $A_{\beta} \subset M_{T, 0}$ after performing the 0 -surgery along $\beta$. To make sure that the two boundary components of $A_{\beta}$ lie in two different component of $R\left(\gamma_{T}\right)$, the suture $\gamma_{T}$ must be arranged as in one of the two possibilities shown in Figure 3. After performing the sutured manifold decomposition along $A_{\beta}$, the new tangle $T_{0}$ has two new $\operatorname{arcs} \alpha_{1}^{\prime}$ and $\alpha_{2}^{\prime}$. For $i=1,2$, let $C_{i}^{\prime}=\partial N\left(\alpha_{i}^{\prime}\right)-\{-1,1\} \times D^{2}$. It is straightforward to check that, after the sutured manifold decomposition along $A_{\beta}$, one and exactly one of the following two possibilities happens, as shown in Figure 3:

- $\gamma^{\prime} \cap C_{1}^{\prime}$ consists of three parallel copies of meridians of $\alpha_{1}^{\prime}$

- $\gamma^{\prime} \cap C_{2}^{\prime}$ consists of three parallel copies of meridians of $\alpha_{2}^{\prime}$.

Without loss of generality, we assume that the first possibility happens, i.e., $\gamma^{\prime}$ contains three copies meridians of $\alpha_{1}^{\prime}$. Removing two such copies, we obtain a new sutured manifold $\left(M_{T_{0}}, \gamma_{T_{0}}\right)$, and by Proposition 2.6 we have

$$
\operatorname{dim}_{\mathbb{Q}} \operatorname{SFH}\left(M_{T_{0}}, \gamma^{\prime}\right)=2 \operatorname{dim}_{\mathbb{Q}} \operatorname{SFH}\left(M_{T_{0}}, \gamma_{T_{0}}\right) .
$$




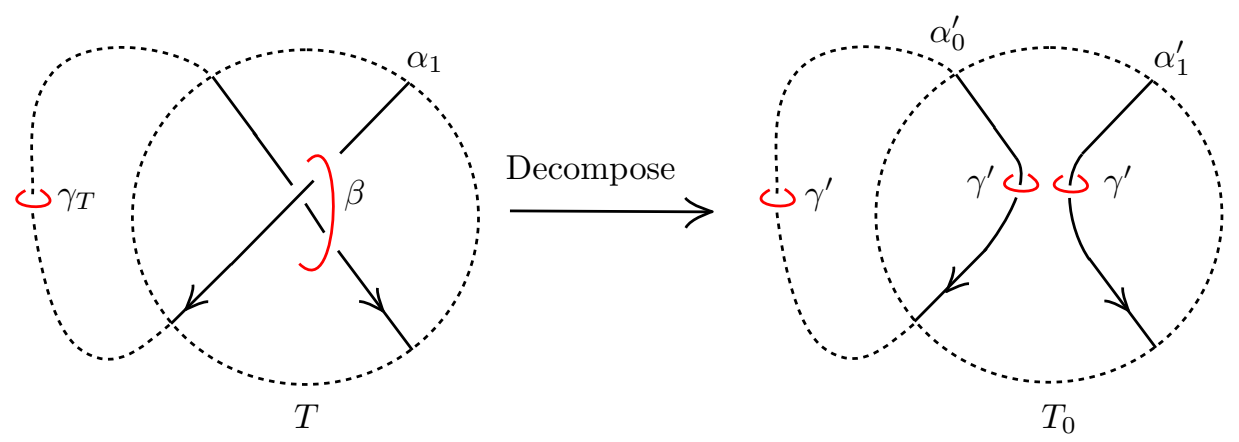

Figure 4. Oriented smoothing

As a result, $\operatorname{dim}_{\mathbb{Q}} \operatorname{SFH}\left(M_{T_{0}}, \gamma^{\prime}\right)$ is even.

Case 2. The crossing linked by $\beta$ involves only one component of $T$. Without loss of generality, we assume it is $\alpha_{1}$, see Figure 4 . Let $C_{i}=\partial N\left(\alpha_{i}\right)-\{-1,1\} \times D^{2}$ for all $i$. To make sure that the two boundary components of $A_{\beta}$ lie in two different components of $R\left(\gamma_{T}\right)$, we must have the suture on $C_{1}$ to be in the position as depicted in Figure 4. After the decomposition along $A_{\beta}$, the new tangle $T_{0}$ now has a closed component, which we call $\alpha_{0}^{\prime}$, and an arc that we call $\alpha_{1}^{\prime}$. Let $C_{0}^{\prime}=\partial N\left(\alpha_{0}^{\prime}\right)$ and $C_{1}^{\prime}=\partial N\left(\alpha_{1}^{\prime}\right)-\{-1,1\} \times D^{2}$. Note that $C_{0}^{\prime}$ is a torus while $C_{1}^{\prime}$ is an annulus. The suture $\gamma^{\prime}$ contains two meridians on $C_{0}^{\prime}$, and one meridian on $C_{1}^{\prime}$ (and one meridian on every other $C_{i}$ ).

Recall that our goal is to show that $\operatorname{dim}_{\mathbb{Q}} \operatorname{SFH}\left(M_{T_{0}}, \gamma^{\prime}\right)$ is even. Write

$$
T_{0}^{\prime}=T_{0} \backslash \alpha_{0}^{\prime} \text {. }
$$

Case 2.1. When $\alpha_{0}^{\prime}$ is split from $T_{0}^{\prime}$, i.e., there is a 3 -ball $B^{3} \subset(-1,1) \times D^{2}$ so that

$$
B^{3} \cap T_{0}=\alpha_{0}^{\prime} .
$$

In this case, we know that $\left(M_{T_{0}}, \gamma^{\prime}\right)$ is a connected sum:

$$
\left(M_{T_{0}}, \gamma^{\prime}\right) \cong\left(M_{T_{0}^{\prime}}, \gamma^{\prime}-B^{3}\right) \#\left(S^{3}\left(\alpha_{0}^{\prime}\right), \gamma^{\prime} \cap B^{3}\right) .
$$

Here $S^{3}\left(\alpha_{0}^{\prime}\right)$ is the knot complement of the knot $\alpha_{0}^{\prime} \subset B^{3} \subset S^{3}$. It then follows from [Juh06, Proposition 9.15] that the dimension of $\operatorname{SFH}\left(M_{T_{0}}, \gamma^{\prime}\right)$ is even.

Case 2.2. When $\alpha_{0}^{\prime}$ is not split from $T_{0}^{\prime}$. Suppse there is a positive crossing of $T_{0}$ involving both $\alpha_{0}^{\prime}$ and $T_{0}^{\prime}$. Pick the circle $\theta$ as depicted in Figure 5. Suppose the component of $T_{0}^{\prime}$ involved in the crossing is $\alpha^{\prime}$. There is a surgery exact triangle associated to $\theta$ :

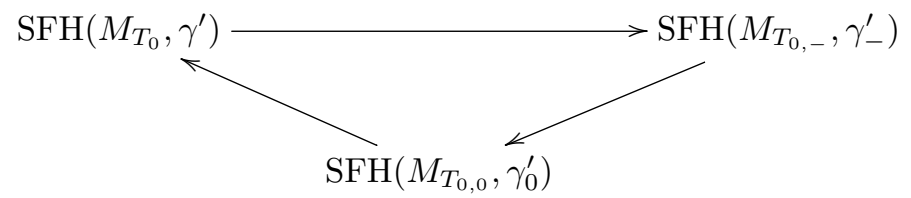

As above, $T_{0,-}$ is obtained from $T_{0}$ by a crossing change and $\left(M_{T_{0,-}}, \gamma_{-}^{\prime}\right)$ is the corresponding sutured manifold. The tangle $T_{0,0}$ is obtained from $T_{0}$ by an oriented smoothing. As in Figure $5, \alpha_{0}^{\prime}$ and $\alpha^{\prime}$ merge into a single component $\alpha_{0}^{\prime \prime} \subset T_{0,0}$. It is then straightforward to check that the new suture $\gamma_{0}^{\prime}$ consists of five meridians of $\alpha_{0}^{\prime \prime}$ : The two meridians of $\alpha_{0}^{\prime}$ and one meridian of $\alpha^{\prime}$ all survive, and there are 

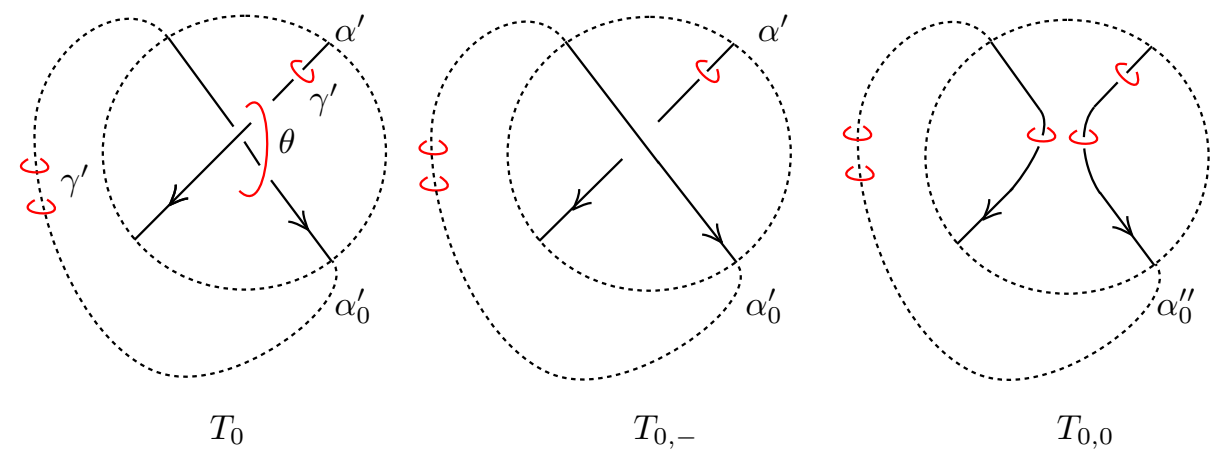

Figure 5. Surgery along $\theta$

two more meridians coming from the decomposition along an annulus $A_{\theta}$ (similar to the annulus $A_{\beta}$ above). By Proposition 2.6, we know that the dimension of $\operatorname{SFH}\left(M_{T_{0,0}}, \gamma_{0}^{\prime}\right)$ is even, and hence

$$
\operatorname{dim}_{\mathbb{Q}} \operatorname{SFH}\left(M_{T_{0}}, \gamma^{\prime}\right) \equiv \operatorname{dim}_{\mathbb{Q}} \operatorname{SFH}\left(M_{T_{0,-}}, \gamma_{-}^{\prime}\right) \bmod 2 .
$$

However, $T_{0}$ and $T_{0,-}$ only differ by a crossing change, and following the same line of Lemma 2.11, there is a finite sequence of such crossing changes that makes $\alpha_{0}^{\prime}$ split from $T_{0}^{\prime}$. Hence, it follows from Case 2.1 and (2.3) that the dimension of $\operatorname{SFH}\left(M_{T_{0}}, \gamma^{\prime}\right)$ must be even. This concludes the proof of Lemma 2.13.

Proof of Proposition 2.8. There is a surgery exact triangle associated to $\beta$ :

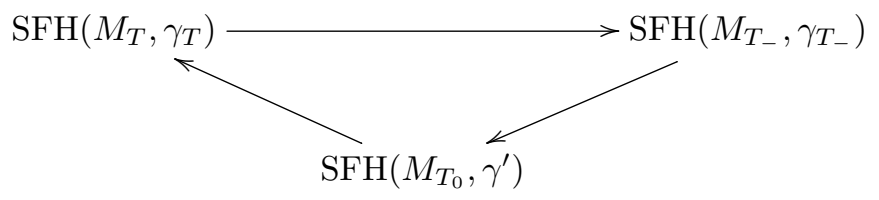

Therefore Lemma 2.13 implies $\operatorname{dim}_{\mathbb{Q}} \operatorname{SFH}\left(M_{T}, \gamma_{T}\right) \equiv \operatorname{dim}_{\mathbb{Q}} \operatorname{SFH}\left(M_{T_{-}}, \gamma_{T_{-}}\right) \bmod 2$. Proposition 2.8 then follows from Lemma 2.9 and Lemma 2.11.

Remark 2.14. The statement and the proof of Proposition 2.8 can be applied to sutured monopole theory and sutured instanton theory as well (with suitable choices of coefficients).

Proof of Proposition 2.7. Take a pair of oppositely oriented meridional sutures to each boundary component of $S^{3}-N(L)$, then $S^{3}-N(L)$ becomes a balanced sutured manifold.

Decompose $S^{3}-N(L)$ along the disk $D$, we obtain a sutured manifold $(M, \gamma)$. The manifold $M$ is given by $[-1,1] \times D^{2}-N(T)$, where $T$ is a tangle in $[-1,1] \times D^{2}$. Since the linking number of $K$ and $U$ is equal to $|K \cap U|$, we have $T=\alpha_{1} \cup \cdots \cup \alpha_{l}$ where $\alpha_{i}$ is an arc from $\{1\} \times D^{2}$ to $\{-1\} \times D^{2}$ for each $i$. The suture $\gamma$ consists of $(l+3)$ components: one meridian on each of $\partial N\left(\alpha_{1}\right), \cdots \partial N\left(\alpha_{l-1}\right)$, three parallel meridians on $\partial N\left(\alpha_{l}\right)$, and one component on $[-1,1] \times \partial D^{2}$ given by $\{\mathrm{pt}\} \times \partial D^{2}$. We have

$$
\widehat{\operatorname{HFL}}\left(L, \frac{l}{2}\right) \cong \operatorname{SFH}(M, \gamma) \text {. }
$$


Removing two sutures from $\partial N\left(\alpha_{l}\right)$, we obtain the sutured manifold $\left(M_{T}, \gamma_{T}\right)$ as in Proposition 2.8. By Proposition 2.6, we have

$$
\operatorname{dim}_{\mathbb{Q}} \operatorname{SFH}\left(M_{T}, \gamma\right)=2 \operatorname{dim}_{\mathbb{Q}} \operatorname{SFH}\left(M_{T}, \gamma_{T}\right) .
$$

Therefore the desired result follows from Proposition 2.8.

\section{Proof of Proposition 1.3}

The strategy of our proof of Proposition 1.3 is to exploit the properties of the multi-variable Alexander polynomial so that we can apply the braid detection property of link Floer homology by Martin [Mar20, Corollary 2]. The link Floer homology and the multi-variable Alexander polynomial are related by (2.1).

Suppose $L$ is a 2 -component link, let $\Delta_{L}(x, y) \in \mathbb{Z}\left[x, y, x^{-1}, y^{-1}\right]$ be the multivariable Alexander polynomial of $L$. Then $\Delta_{L}(x, y)$ is a priori only well-defined up to a multiplication by $\pm x^{a} y^{b}$. It is possible to normalize the Alexander polynomial, for example, using Equation (2.1). However, the Alexander polynomial normalized by (2.1) can be a Laurent polynomial with half-integer exponents. For our purpose, it is more convenient to take $\Delta_{L}(x, y)$ as Laurent polynomial with integer exponents, and therefore we will not normalize $\Delta_{L}(x, y)$.

For $f_{1}, f_{2} \in \mathbb{Z}\left[x_{1}, x_{1}^{-1}, \cdots, x_{n}, x_{n}^{-1}\right]$, we write $f_{1} \doteq f_{2}$ if and only if there exists a multiplicative unit $\epsilon$ such that $f_{1}=\epsilon f_{2}$.

For $f \in \mathbb{Z}\left[x_{1}, x_{1}^{-1}, \cdots, x_{n}, x_{n}^{-1}\right]$, we use $\|f\|$ to denote the sum of the absolute values of the coefficients of $f$. By (2.1), we have

$$
\operatorname{rank}_{\mathbb{Q}} \widehat{\operatorname{HFK}}(L ; \mathbb{Q})=\operatorname{rank}_{\mathbb{Q}} \widehat{\mathrm{HFL}}(L ; \mathbb{Q}) \geq\left\|(1-x)(1-y) \Delta_{L}(x, y)\right\| .
$$

We need the following result.

Theorem 3.1 ([Tor53]). Suppose $L=K_{1} \cup K_{2}$ is a 2-component link with multivariable Alexander polynomial $\Delta_{L}(x, y)$, where $x, y$ are the variables associated to $K_{1}, K_{2}$ respectively. Then we have

$$
\Delta_{L}(x, 1) \doteq \frac{1-x^{l}}{1-x} \Delta_{K_{1}}(x),
$$

where $\Delta_{K_{1}}(x)$ is the Alexander polynomial of $K_{1}$ and $l=1 \mathrm{k}\left(K_{1}, K_{2}\right)$.

From now on, let $L=K \cup U$ be a 2-component link such that

(1) $U$ is an unknot,

(2) $K$ is either a trefoil or an unknot,

(3) the linking number $l=\operatorname{lk}(K, U)$ is positive.

Let $\Delta_{L}(x, y)$ be the multi-variable Alexander polynomial of $L$, where $x, y$ are the variables corresponding to $K$ and $U$ respectively. Define

$$
F(x, y)=(1-x)(1-y) \Delta_{L}(x, y) .
$$

By Theorem 3.1, we have

$$
\Delta_{L}(1, y) \doteq\left(1+y+\cdots+y^{l-1}\right) \Delta_{U}(y)=1+y+\cdots+y^{l-1} .
$$

Write

$$
(1-y) \Delta_{L}(x, y)=: \sum_{m=-\infty}^{+\infty} g_{m}(x) y^{m}
$$


then by definiton,

$$
F(x, y)=\sum_{m}(1-x) g_{m}(x) y^{m}
$$

By (3.1), have

$$
(1-y) \Delta_{L}(1, y) \doteq(1-y)\left(1+y+\cdots+y^{l-1}\right)=1-y^{l} .
$$

Therefore, after multiplying $\Delta_{L}(x, y)$ by $\pm y^{a}$, we may assume without loss of generality that

$$
g_{0}(1)=-g_{l}(1)=1, g_{m}(1)=0 \text { for all } m \neq 0, l .
$$

We establish the following two technical lemmas, which allow us to deduce topological properties of $L$ from the sequence of Laurent polynomials $\left\{g_{m}(x)\right\}_{m \in \mathbb{Z}}$.

Lemma 3.2. Let $L,\left\{g_{m}(x)\right\}_{m \in \mathbb{Z}}$ be as above. If $g_{m}(x)=0$ for all $m \neq 0, l$, then we have $l=1$.

Proof. By the assumption and (3.2),

$$
(1-y) \Delta_{L}(x, y)=g_{0}(x)+g_{l}(x) y^{l} .
$$

Plugging in $y=1$, we have $g_{l}(x)=-g_{0}(x)$, therefore

$$
\Delta_{L}(x, y)=\frac{\left(1-y^{l}\right) g_{0}(x)}{1-y}=\left(1+y+\cdots+y^{l-1}\right) g_{0}(x),
$$

and hence

$$
\Delta_{L}(x, 1) \doteq l g_{0}(x)
$$

On the other hand, by Theorem 3.1,

$$
\Delta_{L}(x, 1) \doteq\left(1+x+\cdots+x^{l-1}\right) \Delta_{K}(x) .
$$

Recall that $l$ is assumed to be positive. Comparing the two equations above, we have

$$
\frac{\Delta_{K}(x)}{l} \in \mathbb{Z}\left[x, x^{-1}\right] .
$$

Since $\Delta_{K}(1)= \pm 1$, this implies $l=1$.

Recall that for a Laurent polynomial $f$, we use $\|f\|$ to denote the sum of the absolute values of the coefficients of $f$.

Lemma 3.3. Let $L,\left\{g_{m}(x)\right\}_{m \in \mathbb{Z}}$ be as above. Suppose the following two conditions hold:

(1) There exists $k \in \mathbb{Z}^{+}$, such that $g_{m}(x)=0$ for all $m \neq 0, l,-k, l+k$,

(2) $\left\|(1-x) g_{0}(x)\right\|=\left\|(1-x) g_{l}(x)\right\|=2$,

then $l=1$ and $K$ is an unknot.

Proof. By Condition (1),

$$
(1-y) \Delta_{L}(x, y)=g_{-k}(x) y^{-k}+g_{0}(x)+g_{l}(x) y^{l}+g_{l+k}(x) y^{l+k} \text {. }
$$

By Condition $(2),\left\|(1-x) g_{0}(x)\right\|=2$. Hence there exists an integer $s>0$ such that $(1-x) g_{0}(x) \doteq 1-x^{s}$, thus

$$
g_{0}(x) \doteq 1+\cdots+x^{s-1} .
$$

By (3.4), this implies $s=1$, therefore $g_{0}(x) \doteq 1$. Similarly $g_{l}(x) \doteq 1$. By (3.4), there exist integers $a, b$, such that

$$
g_{0}(x)=x^{a}, g_{l}(x)=-x^{b} .
$$


Plugging in $y=1$ to $(3.5)$, we obtain

$$
g_{l+k}(x)=x^{b}-x^{a}-g_{-k}(x),
$$

and hence

$$
\begin{aligned}
(1-y) \Delta_{L}(x, y) & =g_{-k}(x) y^{-k}+x^{a}-x^{b} y^{l}+\left(x^{b}-x^{a}-g_{-k}(x)\right) y^{l+k} \\
& =g_{-k}(x)\left(y^{-k}-y^{l+k}\right)+x^{a}\left(1-y^{l+k}\right)-x^{b}\left(y^{l}-y^{l+k}\right) .
\end{aligned}
$$

Therefore

$$
\begin{aligned}
\Delta_{L}(x, 1) & =\lim _{y \rightarrow 1}\left(\frac{g_{-k}(x)\left(y^{-k}-y^{l+k}\right)+x^{a}\left(1-y^{l+k}\right)-x^{b}\left(y^{l}-y^{l+k}\right)}{1-y}\right) \\
& =(l+2 k) g_{-k}(x)+(l+k) x^{a}-k x^{b} .
\end{aligned}
$$

By $(3.7)$, if $\Delta_{L}(x, 1)$ has more than two terms, then at least one of its coefficients is a multiple of $(l+2 k)$.

On the other hand, by Theorem 3.1,

$$
\Delta_{L}(x, 1) \doteq\left(1+x+\cdots+x^{l-1}\right) \Delta_{K}(x),
$$

and hence (recall we have assumed that $K$ is either a trefoil or an unknot)

$$
\Delta_{L}(x, 1) \doteq \begin{cases}1+x+\cdots+x^{l-1} & \text { if } K \text { is an unknot, } \\ 1-x+x^{2} & \text { if } K \text { is a trefoil and } l=1, \\ 1+\sum_{k=2}^{l-1} x^{k}+x^{l+1} & \text { if } K \text { is a trefoil and } l \geq 2 .\end{cases}
$$

In particular, all the coefficients of $\Delta_{L}(x, 1) \in \mathbb{Z}\left[x, x^{-1}\right]$ are \pm 1 . Since $l+2 k \geq 3$, the previous argument implies that $\Delta_{L}(x, 1)$ has at most two terms, and hence there are three possibilities:

(1) $K$ is an unknot, $l=1$,

(2) $K$ is an unknot, $l=2$,

(3) $K$ is a trefoil, $l=2$.

To eliminate the second and third possibilities, notice that in these cases, (3.7) and (3.8) yield

$$
(2 k+2) g_{-k}(x)+(k+2) x^{a}-k x^{b} \doteq x+1 \text { or } x^{3}+1 .
$$

By the assumptions, we have $k \in \mathbb{Z}^{+}$, and hence

$$
(k+2) x^{a}-k x^{b} \doteq x+1 \text { or } x^{3}+1 \quad \bmod (2 k+2),
$$

therefore we have

$$
a \neq b,
$$

and

$$
k+2 \equiv \pm 1, k \equiv \mp 1 \quad \bmod (2 k+2),
$$

which imply $k=1$, thus $g_{-k}(x)=-x^{a}$. This yields a contradiction to (3.4).

Proof of Proposition 1.3. Without loss of generality, we assume $l=\operatorname{lk}(K, U)>0$. By (1.1), we have

$$
\operatorname{dim}_{\mathbb{Q}} \widehat{\operatorname{HFL}}(L ; \mathbb{Q})=\operatorname{dim}_{\mathbb{Q}} \widehat{\operatorname{HFK}}(L ; \mathbb{Q}) \leq 12 .
$$


For $a \in \frac{1}{2} \mathbb{Z}$, we use $\widehat{\mathrm{HFL}}(L, a ; \mathbb{Q})$ to denote the component of $\widehat{\mathrm{HFL}}(L ; \mathbb{Q})$ with degree $a$ on the Alexander grading associated to $U$. Let $\Delta_{L}(x, y), F(x, y), g_{m}(x)$ be as above, and we choose $\Delta_{L}(x, y)$ such that (3.4) holds.

Recall that by $(2.1)$, the coefficients of $F(x, y)$ are the bi-graded Euler characteristics of $\widehat{\operatorname{HFL}}(L ; \mathbb{Q})$. Since $F(1, y)=0$, we have $\operatorname{dim}_{\mathbb{Q}} \widehat{\mathrm{HFL}}(L, a ; \mathbb{Q})$ is even for all $a$. By (2.2), we have

$$
\operatorname{dim}_{\mathbb{Q}} \widehat{\operatorname{HFL}}(L, a ; \mathbb{Q})=\operatorname{dim}_{\mathbb{Q}} \widehat{\operatorname{HFL}}(L,-a ; \mathbb{Q}) .
$$

Since $0 \neq \Delta_{L}(x, y) \doteq \Delta_{L}\left(x^{-1}, y^{-1}\right)$, there is a unique $(a, b) \in \frac{1}{2} \mathbb{Z} \times \frac{1}{2} \mathbb{Z}$, such that $\hat{F}(x, y):=x^{a} y^{b} F(x, y)$ satisfies $\hat{F}(x, y)= \pm \hat{F}\left(x^{-1}, y^{-1}\right)$. Write

$$
\hat{F}(x, y)=\sum_{m \in \frac{1}{2} \mathbb{Z}} \hat{f}_{m}(x) y^{m}
$$

then by (3.4), we have $\hat{f}_{l / 2}(x)= \pm \hat{f}_{-l / 2}\left(x^{-1}\right) \neq 0$. Therefore by (2.1) and (2.2),

$$
\operatorname{dim}_{\mathbb{Q}} \widehat{\operatorname{HFL}}(L, l / 2 ; \mathbb{Q})=\operatorname{dim}_{\mathbb{Q}} \widehat{\operatorname{HFL}}(L,-l / 2 ; \mathbb{Q}) \neq 0 .
$$

Let $s \in \frac{1}{2} \mathbb{Z}$ be the maximum degree such that $\operatorname{dim}_{\mathbb{Q}} \widehat{\mathrm{HFL}}(L, s ; \mathbb{Q}) \neq 0$, then $s \geq l / 2>0$. Since $\operatorname{dim}_{\mathbb{Q}} \widehat{\operatorname{HFL}}(L, s ; \mathbb{Q})$ is even, by $(3.9)$ and $(3.10)$, we have

$$
\operatorname{dim}_{\mathbb{Q}} \widehat{\operatorname{HFL}}(L, s ; \mathbb{Q})=2,4 \text {, or } 6 .
$$

We discuss four cases.

Case 1. $\operatorname{dim}_{\mathbb{Q}} \widehat{\operatorname{HFL}}(L, s ; \mathbb{Q})=2$. By Proposition $2.3, K$ is a braid closure with axis $U$, therefore Case (1) of the proposition holds.

Case 2. $\operatorname{dim}_{\mathbb{Q}} \widehat{\operatorname{HFL}}(L, s ; \mathbb{Q})=4$, and $s=\frac{l}{2}$. By Proposition $2.1, U$ has a Seifert disk that intersects $K$ transversely at $l$ points, therefore this assumption contradicts Proposition 2.7.

Case 3. $\operatorname{dim}_{\mathbb{Q}} \widehat{\mathrm{HFL}}(L, s ; \mathbb{Q})=4$, and $s>\frac{l}{2}$. By $(3.9)$ and $(3.11)$,

$$
\operatorname{dim}_{\mathbb{Q}} \widehat{\operatorname{HFL}}(L, \pm s ; \mathbb{Q})=4, \operatorname{dim}_{\mathbb{Q}} \widehat{\operatorname{HFL}}\left(L, \pm \frac{l}{2} ; \mathbb{Q}\right)=2,
$$

and $\widehat{\operatorname{HFL}}(L, a ; \mathbb{Q})$ vanishes at all the other degrees. By $(2.1),\left\{g_{m}(x)\right\}_{m \in \mathbb{Z}}$ satisfies the assumption of Lemma 3.3, therefore $l=1$ and $K$ is an unknot, and hence Case (2) holds.

Case 4. $\operatorname{dim}_{\mathbb{Q}} \widehat{\mathrm{HFL}}(L, s ; \mathbb{Q})=6$. By $(3.9)$ and $(3.11)$, we have $s=\frac{l}{2}$. By Proposition 2.1, there is a Seifert disk of $U$ that intersects $K$ transversely at $l$ points. By (2.1), $F(x, y)$ is supported at only two degrees in $y$, and hence $\left\{g_{m}(x)\right\}_{m \in \mathbb{Z}}$ satisfies the assumption of Lemma 3.2. By Lemma 3.2, we have $l=1$, therefore $U$ is a meridian of $K$. If $K$ is an unknot, then $L$ is the Hopf link, which satisfies Case (1). Otherwise, $K$ is a trefoil, and hence Case (3) holds.

\section{Proof of the main theorem}

In this section, we prove that Khovanov homology detects the links $L_{1}$ and $L_{2}$ given by Figure 1 .

Recall that the internal grading of the Khovanov homology of a link $L$ is introduced in [BS15, Section 2] as $h-q$, where $h$ is the homological grading, and $q$ is the 
quantum grading. Following [BS15], we use $l$ to denote the internal grading. The next theorem is a special case of a more general result due to Batson and Seed.

Theorem 4.1 ([BS15, Corollary 4.4]). Suppose $L=K_{1} \cup K_{2}$ is a 2-component oriented link. Then we have

$$
\operatorname{rank}_{\mathbb{F}}^{l} \operatorname{Kh}(L ; \mathbb{F}) \geq \operatorname{rank}_{\mathbb{F}}^{l+2} \operatorname{lk}\left(K_{1}, K_{2}\right)\left(\operatorname{Kh}\left(K_{1} ; \mathbb{F}\right) \otimes \operatorname{Kh}\left(K_{2} ; \mathbb{F}\right)\right)
$$

for all $l \in \mathbb{Z}$, where $\mathbb{F}$ is an arbitrary field and rank $^{k}$ denotes the rank of the summand with internal grading $k$.

Let $\mathbb{F}=\mathbb{Z} / 2$ from now on. We have

$$
\operatorname{Kh}\left(U_{2} ; \mathbb{F}\right)=\mathbb{F}_{(-2)} \oplus \mathbb{F}_{(0)}^{2} \oplus \mathbb{F}_{(2)},
$$

where $U_{2}$ is the 2-component unlink and the subscripts denote the internal gradings. Let $T$ be the left-handed trefoil, and let $U$ be the unknot, we have

$$
\mathrm{Kh}(T ; \mathbb{F}) \otimes \mathrm{Kh}(U ; \mathbb{F})=\mathbb{F}_{(0)} \oplus \mathbb{F}_{(2)}^{3} \oplus \mathbb{F}_{(3)} \oplus \mathbb{F}_{(4)}^{3} \oplus \mathbb{F}_{(5)}^{2} \oplus \mathbb{F}_{(6)} \oplus \mathbb{F}_{(7)}
$$

Let $\bar{T}$ be the right-handed trefoil, we have

$$
\mathrm{Kh}(\bar{T} ; \mathbb{F}) \otimes \operatorname{Kh}(U ; \mathbb{F})=\mathbb{F}_{(0)} \oplus \mathbb{F}_{(-2)}^{3} \oplus \mathbb{F}_{(-3)} \oplus \mathbb{F}_{(-4)}^{3} \oplus \mathbb{F}_{(-5)}^{2} \oplus \mathbb{F}_{(-6)} \oplus \mathbb{F}_{(-7)}
$$

Recall that the link $L_{1}=\mathrm{L} 7 \mathrm{n} 1$ can be described as $\hat{\sigma}_{1}^{3} \cup U$ where $\hat{\sigma}_{1}^{3}$ is the closure of the 2-braid $\sigma_{1}^{3}$ with axis unknot $U$, and we choose the orientation as given by Figure 1 and hence the linking number is 2 . The link $L_{2}$ is given by $T \cup U$ where $U$ is a meridian of $T$ and the orientation is chosen so that the linking number is 1 .

We have

$$
\begin{gathered}
\mathrm{Kh}\left(L_{1} ; \mathbb{F}\right)=\mathbb{F}_{(4)} \oplus \mathbb{F}_{(6)}^{3} \oplus \mathbb{F}_{(7)} \oplus \mathbb{F}_{(8)}^{3} \oplus \mathbb{F}_{(9)}^{2} \oplus \mathbb{F}_{(10)} \oplus \mathbb{F}_{(11)}, \\
\mathrm{Kh}\left(L_{2} ; \mathbb{F}\right)=\mathbb{F}_{(2)} \oplus \mathbb{F}_{(4)}^{3} \oplus \mathbb{F}_{(5)} \oplus \mathbb{F}_{(6)}^{3} \oplus \mathbb{F}_{(7)}^{2} \oplus \mathbb{F}_{(8)} \oplus \mathbb{F}_{(9)} .
\end{gathered}
$$

Besides the above links, we define the link $L_{3}=\widehat{\sigma_{1} \sigma_{2}} \cup U$, which is the union of the closure of the 3-braid $\sigma_{1} \sigma_{2}$ and its axis unknot $U$. This is the torus link $T(2,6)$, which is denoted by L6a3 in the Thistlethwaite Link Table. We pick the orientation properly so that the linking number is positive, then

$$
\operatorname{Kh}\left(L_{3} ; \mathbb{F}\right)=\mathbb{F}_{(4)} \oplus \mathbb{F}_{(6)}^{2} \oplus \mathbb{F}_{(7)} \oplus \mathbb{F}_{(8)}^{2} \oplus \mathbb{F}_{(9)}^{2} \oplus \mathbb{F}_{(10)}^{2} \oplus \mathbb{F}_{(11)} \oplus \mathbb{F}_{(12)} .
$$

We now prove Theorem 1.2.

Theorem 1.2. Suppose $L=K_{1} \cup K_{2}$ is a 2-component oriented link and $i \in\{1,2\}$. If $\mathrm{Kh}(L ; \mathbb{F}) \cong \operatorname{Kh}\left(L_{i} ; \mathbb{F}\right)$ (i=1,2) as l-graded abelian groups, then $L$ is isotopic to $L_{i}$ as oriented links.

Proof. Recall that $\mathbb{F}=\mathbb{Z} / 2$. By the assumptions, we have

$$
\operatorname{rank}_{\mathbb{F}} \operatorname{Kh}(L ; \mathbb{F})=12 \text {. }
$$

By [Dow18, Corollary 1.7], we have

$$
\operatorname{rank}_{\mathbb{Q}} \widehat{\operatorname{HFK}}(L ; \mathbb{Q}) \leq 2 \operatorname{rank}_{\mathbb{Q}} \operatorname{Khr}(L ; \mathbb{Q}) \leq 2 \operatorname{rank}_{\mathbb{Z} / 2} \operatorname{Khr}(L ; \mathbb{Z} / 2)=12 .
$$

Theorem 4.1 yields

$$
\operatorname{rank}_{\mathbb{F}} \operatorname{Khr}\left(K_{i} ; \mathbb{F}\right)=\frac{1}{2} \operatorname{rank}_{\mathbb{F}} \operatorname{Kh}\left(K_{i} ; \mathbb{F}\right) \leq \frac{1}{2} \frac{12}{2}=3 .
$$

Therefore $K_{i}(i=1,2)$ is either the unknot or a trefoil according to [KM11, BS18]. By Theorem 4.1 again, we have at least one of $K_{1}$ and $K_{2}$ is the unknot. Without 
loss of generality we assume $K_{2}$ is an unknot, and we discuss two cases.

Case 1. $K_{1}$ is also an unknot. We show that this is contradictory to the assumptions. In fact, Theorem 4.1 and (4.1), (4.4), (4.5) imply that $l=\operatorname{lk}\left(K_{1}, K_{2}\right)$ is no less than 2. Therefore $K_{1}$ is the closure of an $l$-braid with axis $K_{2}$ by Proposition 1.3. Switching the role of $K_{1}$ and $K_{2}$ we obtain that $K_{2}$ is the closure of an $l$-braid with axis $K_{1}$. By Proposition 1.4, we have $l \leq 3$. If $l=2$, then $L=\hat{\sigma}_{1}^{ \pm 1} \cup U$, which is the link L4a1 in the Thistlethwaite Link Table. This contradicts (4.7) because $\operatorname{rank}_{\mathbb{F}} \mathrm{Kh}(\mathrm{L} 4 \mathrm{a} 1 ; \mathbb{F})=8$. If $l=3$, since the only 3 -braid representations of the unknot are given by $\sigma_{1}^{ \pm 1} \sigma_{2}^{ \pm 1}$ and $\sigma_{1}^{ \pm 1} \sigma_{2}^{\mp 1}$, we further divide into two cases:

Case 1.1. $L=\widehat{\sigma_{1} \sigma_{2}} \cup U=L_{3}$ or $L=\widehat{\sigma_{1}^{-1} \sigma_{2}^{-1}} \cup U=\bar{L}_{3}$. Recall that $\operatorname{Kh}\left(L_{3} ; \mathbb{F}\right)$ is given by (4.6). Changing the orientation or taking the mirror image will shift the $l$-grading or change the sign of the $l$-grading, respectively. In any case, the $l$-graded Khovanov homology of $L$ cannot be isomorphic to $\operatorname{Kh}\left(L_{i} ; \mathbb{F}\right)(i \in\{1,2\})$, contradicting the assumptions.

Case 1.2. $L=\widehat{\sigma_{1} \sigma_{2}^{-1}} \cup U$ or $L=\widehat{\sigma_{1}^{-1} \sigma_{2}} \cup U$. In this case $L$ is the link L6a2 (or its mirror image) in the Thistlethwaite Link Table. We have $\operatorname{rank}_{\mathbb{F}} \operatorname{Kh}(L ; \mathbb{F})=20$, which is not the same as $L_{1}$ and $L_{2}$.

In conclusion, $K_{1}$ cannot be an unknot.

Case 2. $K_{1}$ is a trefoil. There are two cases.

Case 2.1. $K_{1}$ is the right-handed trefoil $\bar{T}$. Then Theorem 4.1 and (4.3), (4.4), (4.5) yield a contradiction.

Case 2.2. $K_{1}$ is the left-handed trefoil $T$.

If $\mathrm{Kh}(L ; \mathbb{F}) \cong \operatorname{Kh}\left(L_{1} ; \mathbb{F}\right)$, then by Theorem 4.1 , we have $\operatorname{lk}\left(K_{1}, K_{2}\right)=2$. By Proposition 1.3, the knot $K_{1}$ is the closure of a 2-braid in $S^{3}-N\left(K_{2}\right)$. A 2-braid representing the left-handed trefoil can only be $\sigma_{1}^{3}$. Therefore $L$ is isotopic $L_{1}$.

If $\operatorname{Kh}(L ; \mathbb{F}) \cong \operatorname{Kh}\left(L_{2} ; \mathbb{F}\right)$, then by Theorem 4.1 , we have $\operatorname{lk}\left(K_{1}, K_{2}\right)=1$. By Proposition 1.3 , the knot $K_{2}$ is a meridian of the left-handed trefoil $K_{1}$. Therefore $L$ is isotopic to $L_{2}$.

Remark 4.2. The argument above gives an alternative proof of Martin's theorem that Khovanov homology detects the torus link T(2,6) [Mar20, Theorem 4]. In fact, the link $T(2,6)$ is detected by Case 1.1 in the argument above.

\section{REFERENCES}

[BS15] Joshua Batson and Cotton Seed, A link-splitting spectral sequence in Khovanov homology, Duke Math. J. 164 (2015), no. 5, 801-841. MR3332892

[BS18] John A Baldwin and Steven Sivek, Khovanov homology detects the trefoils, arXiv preprint arXiv:1801.07634 (2018).

[BSX19] John A. Baldwin, Steven Sivek, and Yi Xie, Khovanov homology detects the Hopf links, Math. Res. Lett. 26 (2019), no. 5, 1281-1290. MR4049809

[Dow18] Nathan Dowlin, A spectral sequence from Khovanov homology to knot Floer homology, arXiv preprint arXiv:1811.07848 (2018).

[GL19] Sudipta Ghosh and Zhenkun Li, Decomposing sutured monopole and instanton floer homologies, arXiv preprint arXiv:1910.10842 (2019).

[HN13] Matthew Hedden and Yi Ni, Khovanov module and the detection of unlinks, Geom. Topol. 17 (2013), no. 5, 3027-3076. MR3190305

[Juh06] András Juhász, Holomorphic discs and sutured manifolds, Algebr. Geom. Topol. 6 (2006), 1429-1457. MR2253454 
[Juh08] András Juhász, Floer homology and surface decompositions, Geom. Topol. 12 (2008), no. 1, 299-350. MR2390347

[Juh10] András Juhász, The sutured Floer homology polytope, Geom. Topol. 14 (2010), no. 3, 1303-1354. MR2653728

[Kho00] Mikhail Khovanov, A categorification of the Jones polynomial, Duke Math. J. 101 (2000), no. 3, 359-426. MR1740682

[KM10] P. B. Kronheimer and T. S. Mrowka, Instanton Floer homology and the Alexander polynomial, Algebr. Geom. Topol. 10 (2010), no. 3, 1715-1738. MR2683750

[KM11] Khovanov homology is an unknot-detector, Publ. Math. Inst. Hautes Études Sci. 113 (2011), 97-208. MR2805599

[LS19] Robert Lipshitz and Sucharit Sarkar, Khovanov homology also detects split links, arXiv preprint, arXiv:1910.04246 (2019).

[Mar20] Gage Martin, Khovanov homology detects $T(2,6)$, arXiv preprint, arXiv:2005.02893 (2020).

[Mor85] H. R. Morton, Exchangeable braids, Low-dimensional topology (Chelwood Gate, 1982), 1985, pp. 86-105. MR827298

[OS04] Peter Ozsváth and Zoltán Szabó, Holomorphic disks and knot invariants, Adv. Math. 186 (2004), no. 1, 58-116. MR2065507

[OS08a] _ Holomorphic disks, link invariants and the multi-variable Alexander polynomial, Algebr. Geom. Topol. 8 (2008), no. 2, 615-692. MR2443092

[OS08b] _ Link Floer homology and the Thurston norm, J. Amer. Math. Soc. 21 (2008), no. 3, 671-709. MR2393424

[Ras03] Jacob Andrew Rasmussen, Floer homology and knot complements, ProQuest LLC, Ann Arbor, MI, 2003. Thesis (Ph.D.)-Harvard University. MR2704683

[Sar11] Sucharit Sarkar, A note on sign conventions in link Floer homology, Quantum Topol. 2 (2011), no. 3, 217-239. MR2812456

[Shu14] Alexander N. Shumakovitch, Torsion of Khovanov homology, Fund. Math. 225 (2014), no. 1, 343-364. MR3205577

[Tor53] Guillermo Torres, On the Alexander polynomial, Ann. of Math. (2) 57 (1953), 57-89. MR52104

[XZ19] Yi Xie and Boyu Zhang, Classification of links with khovanov homology of minimal rank, arXiv preprint arXiv:1909.10032 (2019).

[XZ20] _ On links with Khovanov homology of small ranks, arXiv preprint arXiv:2005.04782 (2020).

Department of Mathematics, Massachusetts Institute of Technology, Massachusetts 02139, USA

E-mail address: zhenkun@mit.edu

Beijing International Center for Mathematical Research, Peking University, BeiJING 100871, CHINA

E-mail address: yixie@pku.edu.cn

Department of Mathematics, Princeton University, New Jersey 08544, USA

E-mail address: bz@math.princeton.edu 